\title{
EL TEMBLOR DE QUEPOS DEL 20 DE AGOSTO DE 1999 (6,9 Mw): FUENTE SÍSMICA Y EVOLUCIÓN DE LA SISMICIDAD
}

\author{
Wilfredo Rojas \& Carlos Redondo \\ Sección de Sismología y Vulcanología, Escuela Centroamericana de Geología, \\ Universidad de Costa Rica. Apdo 214-2060. E-mail: wrojas@geologia.ucr.ac.cr \\ credondo@geologia.ucr.ac.cr
}

(Recibido 13/9/01; Aceptado 6/5/02)

\begin{abstract}
The seismic sequence began on august 20, 1999 at 04:02 with an event of 6.9 Mw (seismic moment magnitude), located $50 \mathrm{~km}$ south of Quepos and a maximum intensity of VII (MM). Immediately the main shock was followed by 500 aftershocks of magnitude up to $2.5 \mathrm{ML}$ within the first 24 hours. During the second day after the main shock the activity quickly decayed to an average of 5 events by day. On august 21, 1999 at 04:49 LT, took place an event of 5,6 Mw with an intensity of V (MM) in Quepos and IV in San José, which is considered the major aftershock of the sequence.

Seismic wave analysis by SEISAN computer program and macro seismic data indicated that the rupture area had an extension of $1000 \mathrm{~km} 2$ and a displacement of $43 \mathrm{~cm}$. The seismicity was related to two different seismic sources, a pure subduction process and an asperity by a submarine mountain on the Cocos plate. The main shock was a characteristic subduction event and the second was an upper crustal event, related with a strike slip fault.
\end{abstract}

RESUMEN: La secuencia sísmica se inició el 20 de agosto de 1999 a las 04:02 a.m., con un fuerte sismo ocurrido a $50 \mathrm{~km}$ al sur de Quepos, con una magnitud estimada con base en el momento sísmico, de 6,9 (Mw). Fue seguido por unos 500 temblores de magnitud ML mayor a 2,5 durante las primeras 24 horas hasta decaer durante los 30 días posteriores, a un número de unos 5 diarios de ML superior a 2,5. El día 21/08/99 a las 04:49 a.m., ocurrió el segundo evento de mayor magnitud 5,6 Mw, registrando intensidad V en Quepos y IV en San José. Con la interpretación, el análisis de las señales de ondas sísmicas del evento principal y las réplicas, mediante el programa de cómputo SEISAN y de la información macrosísmica recolectada, se obtuvo que el área de ruptura del evento principal fue de en unos $1000 \mathrm{~m} 2$ y el desplazamiento en el plano de deslizamiento de $43 \mathrm{~cm}$.

La evolución de la sismicidad inmediata ocurrió principalmente en dos fuentes sismogeneradoras de diferente naturaleza, pero asociadas con una aspereza o monte submarino ubicado al sur de Quepos. Por consiguiente, la actividad se inició con un evento característico de subducción y pocas horas después tuvo lugar otra actividad relacionada con un sistema de fracturamiento transcurrente de la parte superior de la corteza.

\section{INTRODUCCIÓN}

El evento sísmico del 20 de agosto de 1999 (Ms 6,9, Mw 6,9) fue sentido en todo el país, provocando algunos daños en los poblados de la cos- ta pacífica central de Costa Rica generando alarma y preocupación entre los pobladores. Sin embargo, no se registraron víctimas fatales y daños mayores en la infraestructura, debido a que el hipocentro se ubicó bajo el piso oceánico y a una 
distancia de $50 \mathrm{~km}$ de Quepos, el principal centro de población, cuyo basamento está constituido por roca firme, que favorece la atenuación de las ondas sísmicas.

Frente a Quepos se subduce la placa Coco bajo la placa Caribe, en cuyo plano de interacción se presentan varios promontorios submarinos que provocan diversos efectos geológicos en la corteza y características particulares de la sismicidad, lo cual se trata de explicar en este estudio.

Los resultados del análisis de la secuencia sísmica se han integrado con los recientes datos del proyecto PAGANINI dirigido por GEOMAR, Alemania, dando como resultado que la sismicidad de la zona estudiada está directamente relacionada con promontorios submarinos que han sido subducidos frente a la costa pacífica central de Costa Rica, comprobando una vez más la existencia de asperezas en la zona de Benioff, que da lugar a una fuente sísmica muy puntual, localizada unos $50 \mathrm{~km}$ al Sur de Quepos y que genera otros efectos en la parte superior de la placa cabalgante (Caribe).

\section{CARACTERÍSTICAS DE LA ZONA SÍSMICA DE QUEPOS}

Originalmente fue definida por Morales (1985); posteriormente Rojas et al., (1998) la denominan como fuente sísmica de subducción Quepos - Sierpe. Se ubica en el margen convergente de Costa Rica, frente a la costa pacífica central, extendiéndose desde Punta Judas hasta la desembocadura del río Sierpe. Esta zona presenta una orientación de esfuerzos compresivos $\mathrm{N} 30^{\circ}-40^{\circ} \mathrm{E}$ y un ángulo de buzamiento promedio de $30^{\circ}$ al NE y los sismos alcanzan profundidades máximas del orden de los 100 km (Montero, 1986; Barquero \& Climent, 1999).

No se ha establecido aun la recurrencia sísmica de esta fuente pero tiene capacidad de generar eventos importantes, como los ocurridos el 03/03/1882 o el 09/09/1952, ambos de magnitud 7,0 Ms (Montero \& Climent, 1990), que provocaron importantes daños en el Valle Central. A lo largo de la zona costera del pacífico central de Costa Rica, esta fuente sísmica podría generar intensidades máximas de VIII.
En el perfil de la línea sísmica de reflexión Sonne No. 81-linea 6 (Ye et al., 1996), se observa la presencia de un monte submarino en las coordenadas $08^{\circ} 50^{\prime}$ y $-84^{\circ} 40^{`}$ y una serranía submarina subducida. Este promontorio se ubica unos $75 \mathrm{~km}$ al sur de Quepos y se extiende hacia el noreste, hasta una zona aún no determinada en los perfiles de la sísmica de reflexión.

\section{ANÁLISIS DE LOS DATOS SISMOLÓGICOS E INTERPRETACIÓN}

Para efectuar el análisis de la información sismológica ligada a la secuencia sísmica, se procedió a instalar dos estaciones sismográficas portátiles. La zona donde ocurrió la secuencia sísmica está relativamente bien cubierta por los instrumentos sismográficos de la RSN (ICE-UCR), la cual opera estaciones permanentes en Quepos, Golfito y Lajas (cerca de Jacó). El proceso de localización de los sismos, se efectuó utilizando un modelo de corteza híbrido de 5 capas, donde las dos primeras capas superficiales fueron las obtenidas por Ye et al. (1996) para la zona de Dominical. Sin embargo, para las capas más profundas (cercanas al Moho), se utilizó el nuevo modelo propuesto para el sur de Costa Rica por Leandro $\&$ Rojas (2001). Lo anterior, debido a que el modelo de Ye et al. (1996) presenta una capa de baja velocidad entre los 15 a $30 \mathrm{~km}$, que originaba altos residuos en el proceso de localización de los sismos réplicas ocurridos.

El estudio de campo se inició el día del sismo principal, con la instalación de una estación portátil digital en Hatillo de Dominical y otra en bahía Drake. La primera presentó problemas de registro por daños en el control de tiempo del GPS, sin embargo, con la estación de Drake se logró registrar varias réplicas y sus datos se integraron en las localizaciones.

Los datos de campo recopilados (Cuadro 1) ayudaron a la elaboración del mapa de intensidades (Fig. 1), en el que se refleja una concentración de los mayores efectos, hacia el norte de la zona mesosísmica, específicamente en el área que comprende los poblados de Dominical, Tinamaste, Tumbas y Platanillo. En este último lugar, 
Cuadro 1

Registro de intensidades y efectos del sismo del 20/08/99 04:02 AM (6,9 Mw)

\begin{tabular}{|c|c|c|}
\hline LUGAR & EFECTOS & INTENSIIDAD M.M \\
\hline $\begin{array}{l}\text { Tumbas } \\
\text { Tinamaste } \\
\text { Platanillo }\end{array}$ & $\begin{array}{l}\text { Derrumbes en laderas, deslizamientos en los caminos, } \\
2 \text { casas severamente dañadas en Tinamaste de Pérez Zeledón. }\end{array}$ & VII \\
\hline $\begin{array}{l}\text { Quepos } \\
\text { San Pablo (León Cortés) } \\
\text { Dominical } \\
\text { San Isidro del General }\end{array}$ & $\begin{array}{l}\text { Caída de estantes y objetos, agrietamientos en paredes } \\
\text { y pisos. Derrumbes en laderas. } \\
\text { Caída de repellos en San Isidro del General. } \\
\text { Varias réplicas sentidas. }\end{array}$ & VI \\
\hline $\begin{array}{l}\text { San Ignacio de Acosta } \\
\text { Orotina } \\
\text { San José } \\
\text { Atenas } \\
\text { Turrialba } \\
\text { Palmares } \\
\text { Puriscal }\end{array}$ & $\begin{array}{l}\text { Personas salieron a la calle. } \\
\text { Derrumbes en montes del Aguacate, agrietamiento } \\
\text { menor en casas en Acosta. } \\
\text { Duró } 15 \text { segundos la fase sentida, vibración fuerte de } \\
\text { ventanales,caída menor de rocas en la carretera } \\
\text { Atenas-Alajuela. } \\
\text { Se quebraron botellas en negocios del centro de San José. }\end{array}$ & $\mathrm{V}$ \\
\hline $\begin{array}{l}\text { Golfito } \\
\text { Jacó } \\
\text { San Ramón }\end{array}$ & $\begin{array}{l}\text { Muy fuerte, gente salió de sus casas, se fue la luz, } \\
\text { algunos objetos cayeron. }\end{array}$ & IV-V \\
\hline $\begin{array}{l}\text { Las Juntas } \\
\text { Puntarenas }\end{array}$ & & \\
\hline $\begin{array}{l}\text { Cóbano } \\
\text { Guápiles } \\
\text { Bataan } \\
\text { Bribrí } \\
\text { Puerto Armuelles, Panamá } \\
\text { Boquete, Panamá } \\
\text { David, Panamá }\end{array}$ & $\begin{array}{l}\text { Fuerte a más o menos fuerte, despertó a muchas personas, } \\
\text { vibraron los techos. Daños leves en paredes de casas } \\
\text { de baja calidad en Chiriquí, Panamá, donde las loras } \\
\text { saltaron de los árboles al suelo. }\end{array}$ & IV \\
\hline $\begin{array}{l}\text { Liberia } \\
\text { Penonomé, Panamá }\end{array}$ & Sentido por gran parte de la población, no hay daños & III-IV \\
\hline $\begin{array}{l}\text { Upala } \\
\text { Los Chiles } \\
\text { Barra del Colorado }\end{array}$ & $\begin{array}{l}\text { Sentido por algunas personas. } \\
\text { Leve no hay daños. }\end{array}$ & III \\
\hline $\begin{array}{l}\text { La Cruz } \\
\text { San Juan del Sur, Nicaragua } \\
\text { Playa del Coco }\end{array}$ & $\begin{array}{l}\text { Muy leve, solamente muy pocas personas lo sintieron. } \\
\text { En San Juan del Sur, Nicaragua, se sintió una } \\
\text { sensación de mareo. }\end{array}$ & II \\
\hline
\end{tabular}

una vivienda sufrió serios daños en los cimientos por falseamiento del terreno y otra sufrió daño parcial en las paredes.

Se presentaron deslizamientos en los cortes de camino entre Tinamaste y Platanillo, bloqueando el tránsito por varias horas. En Domini- cal, Hatillo de Aguirre y Quepos, varias casas y negocios sufrieron agrietamientos en sus paredes. Se observaron deslizamientos en las laderas de la fila Cariblanco, al norte de Dominical. La intensidad estimada en esta zona es de VII en la escala Mercalli Modificada (Fig. 1). 


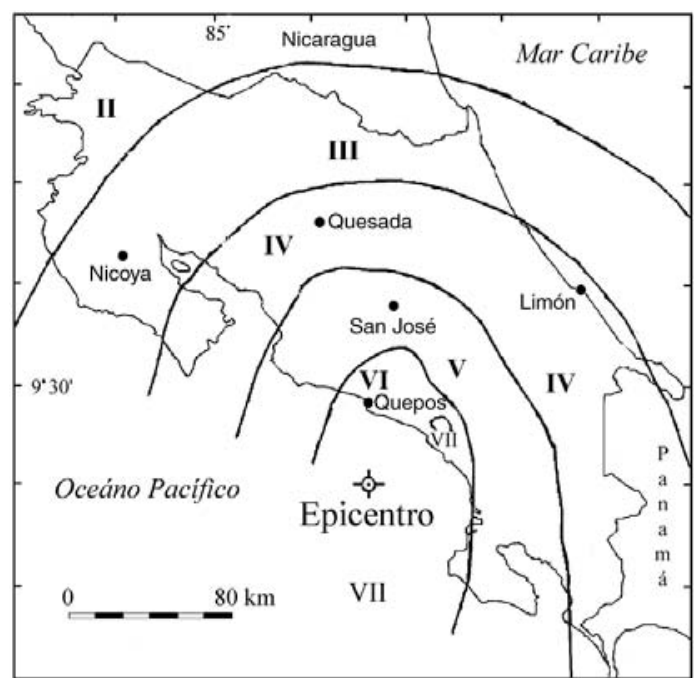

Fig. 1: Mapa de intensidades para el terremoto de Quepos del 20/08/99 (6,9 Mw). Los círculos rellenos, corresponden a lugares donde se determina los valores de intensidad.

El Centro Sismológico de América Central (CASC) localizó el sismo, usando datos de 43 estaciones sísmicas, incluyendo 6 registros de banda ancha, asignándole una magnitud $6,7 \mathrm{Ms}$ y $6,6 \mathrm{Mw}$ y ubicando el epicentro a $40 \mathrm{~km}$ sur de Quepos a $24 \mathrm{~km}$ de profundidad.

El valor de aceleración máxima medido en la estación ISD, ubicada en San Isidro del General, fue de $0,8 \mathrm{~m} / \mathrm{s}^{2}$ y en San José centro de 0,31 $\mathrm{m} / \mathrm{s}^{2}$ (Schmidt et al., 1999). El último valor concuerda bastante bien con el estimado para San José, de acuerdo con la curva de atenuación local de Schmidt et al. (1997). Ambos valores de aceleración no reflejan una fuerte sacudida, capaz de producir daño importante a las estructuras.

La secuencia sísmica es del tipo evento principal y réplicas, iniciando con un fuerte temblor el día 20/08/99 a las 04:02 hora local, seguido por una gran cantidad de sismos de magnitudes menores, donde muchas de ellas fueron réplicas y otros resultado de la reactivación de una fuente sísmica aledaña. En esta última se generó once horas después (el día 21 a las 04:49Lt) el segundo evento de mayor tamaño con magnitud $5,6 \mathrm{Mw}$ y que fue ubicado a unos $20 \mathrm{~km}$ al este del epicentro del evento principal a una profun- didad de 3,5 km. Aproximadamente 500 sismos de magnitudes mayores 2,5 Ml fueron registradas en las primeras 24 horas. La actividad se prolongó hasta inicios de setiembre, disminuyendo en magnitud y números de eventos. La figura 2 resume la evolución de la sismicidad durante las primeras 36 horas desde el evento principal, para sismos de magnitudes mayores a 3,5 Ml.

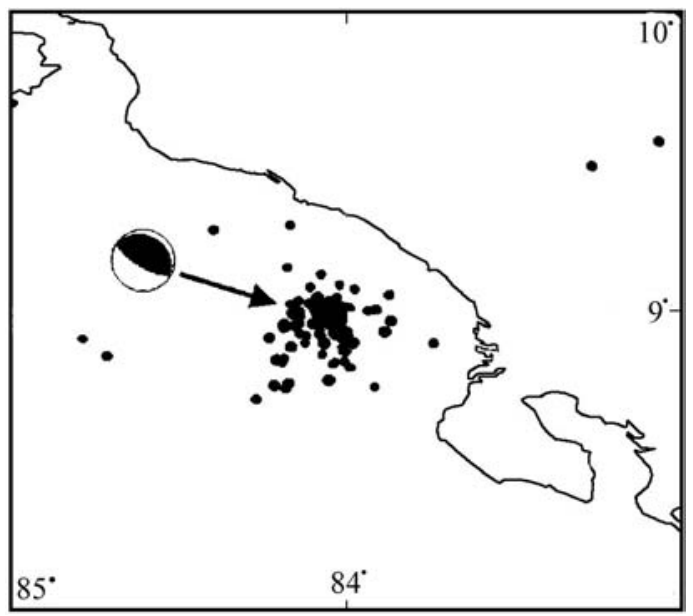

Fig. 2: Mapa de sismicidad ( $\mathrm{M}>3.5$ ) del 2 al 23/08/99, posterior al sismo de Quepos del 20 de agosto de 1999, obsérvese el mecanismo focal inverso. Eventos totales: 77.

Se observó que en los primeros tres días de actividad presentó un comportamiento migratorio en espacio y en el tiempo. Se definen dos principales fuentes sísmicas, una por subducción y otra por fallamiento superficial; relacionadas ambas por una misma causa, que se relaciona con la serranía submarina sumergida frente a Quepos. La actividad sísmica inicial se dio por subducción predominantemente entre los 20 a $30 \mathrm{~km}$ de profundidad, especialmente durante las primeras cinco horas, donde los mecanismos focales son principalmente del tipo inverso, con deslizamiento a lo largo del plano de Benioff. Posteriormente ocurrió una actividad sísmica en la parte superior de la placa Caribe, concentrada entre los 5 a $10 \mathrm{~km}$ de profundidad, cuyos epicentros se alinearon en dirección N-NE a lo largo de aproximadamente $15 \mathrm{~km}$ y separados unos $10 \mathrm{~km}$ al este del epicentro del evento principal. El análisis 
de los mecanismos focales de estos eventos generados en la corteza superior indican un proceso de ruptura, predominantemente de tipo lateral derecho (Fig. 3).

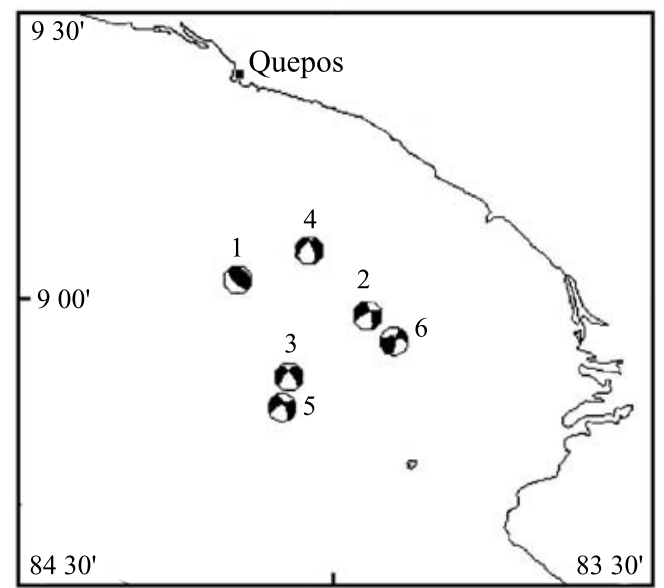

Fig. 3: Mapa con las figuras de los principales mecanismos focales. El No 1 es el evento principal y corresponde a fallamiento inverso.

Anteriormente, en el mapa geodinámico de Costa Rica de Montero et al. (1990) se habían registrado varios sismos de la zona con ese proceso de ruptura. Además, en el perfil sísmico Sonne No. 81 Línea 6 (Ye et al., 1996), se observa un sistema de fallamiento en la parte superior de la corteza, hacia la parte este del levantamiento de Quepos, que coincide con lo obtenido en los mecanismos focales de los eventos superficiales de esa área (Fig. 3).

\section{PARÁMETROS CINEMÁTICOS Y DINÁMICOS DEL PROCESO DE RUPTURA DEL SISMO PRINCIPAL}

El análisis del mecanismo focal efectuado por la Red Sismológica Nacional (RSN) se efectuó con los datos de polaridad de 18 estaciones sismográficas locales, obteniendo una solución prácticamente similar a la reportada por el Servicio Geológico de Estados Unidos de Norteamérica (USGS). Se trata de una falla inversa pura, con rumbo de $315^{\circ}$ y buzamiento $20^{\circ} \mathrm{NE}$ (Fig. 3). El momento sísmico reportado por la Universidad de Harvard (HVR) en los Estados Unidos, fue:

$$
\mathrm{Mo}=2,6 \times 10^{19} \mathrm{Nm}
$$

La magnitud del momento sísmico, determinado con base en el análisis espectral de la señal registrada en la componente vertical de la estación de período medio de la Universidad de Costa Rica, fue de 6,6 Mw y el de la Universidad de Harvad fue de 6,9 Mw. Se asumió un valor del módulo de rigidez de $\mu=6 \times 10^{11}$ dinas $/ \mathrm{cm}^{2}$, que es lo recomendado para regiones corticales en arcos de islas, muy similares a las condiciones de Costa Rica.

El área de ruptura se determinó a partir de las réplicas originadas a profundidades mayores a $20 \mathrm{~km}$, ocurridas entre el 20 y 23 de agosto (Fig. 3 ), definiéndose una zona de $25 \times 40 \mathrm{~km}$, correspondiendo a un área de ruptura $\mathbf{A}=1000 \mathrm{~km}^{2}$.

A partir de los valores anteriores se estima u, el desplazamiento a lo largo del plano de ruptura, de acuerdo con la relación

$$
\mathrm{Mo}=\mu \mathrm{uA}
$$

Se determinó que el desplazamiento u a lo largo de la ruptura es de $43 \mathrm{~cm}$ y considerando la velocidad promedio de subducción de la placa Coco $(9 \mathrm{~cm} / \mathrm{año})$, un evento de este tipo, tiende a liberar la energía que se acumula en la zona durante unos cinco años. Lo anterior indica que existe un alto porcentaje de deslizamiento asísmico (90\%), considerando que sismos de magnitudes cercanas a 7,0 ocurren cada 30 o 40 años en esta zona.

\section{CONSIDERACIONES GENERALES}

El evento sísmico de Quepos, fue el sismo de mayor tamaño ocurrido en América Central durante el año 1999. Alcanzó una intensidad de VII en los poblados cercanos a Dominical, como Tinamaste, Tumbas y Platanillo.

Las características sismológicas y solución de mecanismo focal indican que el sismo principal fue un evento típico de subducción ocurrido en la zona de interacción de las placas Coco y Caribe y relacionado con una aspereza o montaña submarina localizada unos $45 \mathrm{~km}$ al sur de Quepos. 
La sismicidad, mecanismos focales $y$ perfiles de sísmica de reflexión indican la existencia de un sistema de ruptura superficial, en dirección aproximadamente NNE, dentro de la parte superior de la placa Caribe, que fue reactivado pocas horas después del evento principal y que parece estar asociada al borde este de la serranía submarina antes mencionada. Por consiguiente, ocurrió un movimiento combinado entre fallamiento inverso por subducción y ruptura cortical de desplazamiento de rumbo en la parte superior de la corteza.

El área de ruptura asociada al evento principal se estima en $1000 \mathrm{~km}^{2}$ y el desplazamiento a lo largo del plano de ruptura de unos $43 \mathrm{~cm}$.

\section{AGRADECIMIENTOS}

A Luis Fernando Brenes y Héctor Flores por su apoyo en el estudio de campo y a Keneth Pérez por la preparación de las figuras.

\section{REFERENCIAS}

BARQUERO, R. \& CLIMENT, A., 1999: Los temblores de la zona de Quepos de agosto de 1999, fuente sísmica y características tectónicas. - 27 págs. Unidad de Amenaza y Auscultación Sismovolcánica, ICE [Inf. técnico].
LEANDRO, G. \& ROJAS, W., 2001: Modelo de corteza de la región de Boruca, Valle del Diquís.- 49 págs. ICEUCR, [Inf. técnico].

MONTERO, W. \& CLIMENT, A., 1990: Los terremotos de Grecia de 1882 y de Quepos de 1952, Costa Rica: distribución de intensidades y origen tectónico. Ciencia y Tecnología, 14 (1-2): 81-105.

MONTERO, W., PANIAGUA, S., KUSSMAUL, S. \& RIVIER, F., 1990: Geodinámica interna de Costa Rica. - Rev. Geol. América Central, 14: 1-12.

MONTERO, W., 1986: Períodos de recurrencia y tipos de secuencias sísmicas de los temblores interplaca e intraplaca en la región de Costa Rica. - Rev. Geol. América Central, 5: 35-72.

ROJAS, W,. LINDHOLM, C. \& BUNGUM, H., 1998: Seismic hazard analisis for the Metropolitan Area of the Central Valley, Costa Rica. - 59 págs. NORSAR, Kjeller [Inf. técnico].

SCHMIDT, V., DAHLE, A. \& BUNGUM, H. 1997: Costa Rican spectral strong motion attenuation. - 45 págs. NORSAR, Kjeller [Inf. técnico].

SCHMIDT, V., ARÓN, C. \& SEGURA, C., 1999: Registros del sismo del 10/08/99 por los acelerógrafos digitales del Lab. de Ing. Sísmica de UCR.- 5 págs. Univ de Costa Rica, San José [Inf. técnico].

YE, S., BIALAS, J., FLUEH, E., LEANDRO, G., HINZ, K., STAVENHAGEN, A., \& VON HUENE, R., 1996: Crustal structure of Middle América trench of Costa Rica from wide-angle seismic data. - Tectonics, 15 (5): $1006-1021$. 